Tropical Journal of Pharmaceutical Research February 2020; 19 (2): 259-264

ISSN: $1596-5996$ (print); 1596-9827 (electronic)

(C) Pharmacotherapy Group, Faculty of Pharmacy, University of Benin, Benin City, 300001 Nigeria.

Available online at http://www.tjpr.org

Original Research Article

http://dx.doi.org/10.4314/tjpr.v19i2.7

\title{
Evaluation of chidamide and PFI-1 as a combination therapy for triple-negative breast cancer
}

\author{
Nong Lin ${ }^{1 *}$, Qiaolu Yang ${ }^{1}$, Tong $\mathrm{Xu}^{2}$, Lianguo Shi ${ }^{3}$ \\ ${ }^{1}$ Department of Breast Surgery, ${ }^{2}$ Department of Internal Medicine-Oncology, ${ }^{3}$ Department of Pathology, First Affiliated Hospital \\ of Xiamen University, Xiamen City, Fujian Province 361000, China
}

*For correspondence: Email: NongLinfjk@163.com; Tel: +86-592-2137512

Sent for review: 7 November 2019

Revised accepted: 30 January 2020

\begin{abstract}
Purpose: To evaluate the in vitro and in vivo effects of the combination therapy of histone deacetylases (HDAsC) inhibitor, chidamide, and bromodomain-containing proteins (BETs) inhibitor, PFI-1, on triplenegative breast cancer (TNBC).

Methods: Four distinct breast cancer cell lines and one TNBC mouse model were treated with vehicle, chidamide, PFI-1 alone, or chidamide and PFI-1. The inhibitory effect of chidamide or PFI-1 on HDACs and BETs was assessed by HDAC enzyme inhibition and AlphaScreen assays. Cell viability was determined by MTT assay while protein expression of p-STAT3 was evaluated by western blotting and immunohistochemistry $(\mathrm{IHC})$ staining assay.

Results: Chidamide exerted inhibitory effect on HDACs while PFI-1 inhibited BET proteins. The threedimensional model demonstrated the interactions between chidamide and HDAC2, and between PFI-1 and BRD4. Chidamide or PFI-1 exerted inhibitory effects on breast cancer cell proliferation in vitro. However, the combination of PFI-1 and chidamide significantly inhibit MDA-MB-231 cell viability, and decrease the expression of p-STAT3, when compared to that treated with chidamide or PFI-1 alone. Moreover, the combined inhibitory effect of PFI-1 and chidamide on tumor growth was also found in the in vivo mice experiments.

Conclusion: The combination of chidamide and PFI-1 is a potential is a potential therapeutic strategy for the management of TNBC.
\end{abstract}

Keywords: Triple-negative breast cancer, Histone deacetylases, Bromodomain

This is an Open Access article that uses a fund-ing model which does not charge readers or their institutions for access and distributed under the terms of the Creative Commons Attribution License (http://creativecommons.org/licenses/by/4.0) and the Budapest Open Access Initiative (http://www.budapestopenaccessinitiative.org/read), which permit unrestricted use, distribution, and reproduction in any medium, provided the original work is properly credited.

Tropical Journal of Pharmaceutical Research is indexed by Science Citation Index (SciSearch), Scopus, International Pharmaceutical Abstract, Chemical Abstracts, Embase, Index Copernicus, EBSCO, African Index Medicus, JournalSeek, Journal Citation Reports/Science Edition, Directory of Open Access Journals (DOAJ), African Journal Online, Bioline International, Open-J-Gate and Pharmacy Abstracts

\section{INTRODUCTION}

Triple-negative breast cancer (TNBC), with poor prognosis and high rate of metastasis, is a heterogeneous breast cancer subtype [1] that accounts for approximately $20 \%$ of newly diagnosed primary breast cancers [2]. Current therapeutic options for TNBC are limited to cytotoxic therapies, and there are no approved targeted therapies [3-5]. Current progress in biochemical and technological research has identified key pathways and proteins that could be used as potential therapeutic targets for TNBC treatments [6-8].

Epigenetic mechanisms are increasingly considered as primary contributors to the 
pathogenesis of cancers such as lymphoma[9], pancreatic ductal adenocarcinoma, and melanoma[10]. Histone deacetylases (HDACs) could regulate epigenetic genes through the modification of histone tails. HDAC inhibitors (HDACi) represent the most promising epigenetic mechanism-based malignant tumor therapy [11]. Many $\mathrm{HDACi}$, including chidamide and vorinostat, are used as potential therapeutic approaches for T-cell lymphoma and multiple myeloma [12].

The bromodomain and extraterminal (BET) motif family of proteins, which include BRDT, BRD4, BRD3, and BRD2, specifically recognize acetylated lysine residues on histones and other proteins, such as chromatin, to alter their cellular epigenetic and transcriptional programs [13]. Small molecule BET inhibitors (BETi) such as PFI-1 and JQ1, suppress the expression of oncogenes, leading to the inhibition of cell growth and cell apoptosis. Recently, a study reported synergistic effects of $\mathrm{HDACi}$ and $\mathrm{BETi}$ in lymphoma, urothelial carcinoma, , non-small cell lung cancer, and particularly, breast cancer [14]. In this study, the synergistic effects of the HDACi chidamide and the BETi PFI-1 used as a combination therapy in TNBC were investigated.

\section{EXPERIMENTAL}

\section{Molecular modeling}

The computational docking program, Discovery Studio 3.0, was used to dock chidamide to HDAC2. The receptor was prepared using the Protein Preparation Wizard in the Schrödinger suite, and the ligands were initially minimized using the LigPrep module. The Glide module was employed for the docking study.

\section{Compounds}

Chidamide and PFI-1 were purchased from Selleck Chemicals (https://www.selleck.cn/) and dissolved in dimethyl sulfoxide (DMSO) to yield $10 \mathrm{mM}$ stock solutions.

\section{HDAC enzyme inhibition assay}

The HDAC enzyme inhibition assay was completed and analyzed using human recombinant HDAC proteins. The base reaction buffer was composed of $137 \mathrm{mM} \mathrm{NaCl}, 50 \mathrm{mM}$ Tris- $\mathrm{HCl}(\mathrm{pH} 8.0), 2.7 \mathrm{mM} \mathrm{KCl}$, and $1 \mathrm{mM} \mathrm{MgCl} 2$ to which was added fresh $1 \mathrm{mg} / \mathrm{mL}$ BSA and $1 \%$ DMSO. Deacetylation was performed by the addition of $2 x$ enzyme in wells of the reaction plate, into which buffer was added. The compounds in $100 \%$ DMSO were then preincubated with the enzyme mixture using acoustic technology (Echo550; nanoliter range). The substrate mixture was added to reaction wells. The plates were sealed, rotated, shaken, and incubated for $2 \mathrm{~h}$ at $30^{\circ} \mathrm{C}$. The developer containing trichostatin $\mathrm{A}$ (or nicotinamide for SIRTs) was then added and a fluorescent signal was generated. Kinetic measurements were then taken after $1.5 \mathrm{~h}$ using Envision system at $15-\mathrm{min}$ intervals (excitation/emission $=360 / 460 \mathrm{~nm}$ ). When the development of the fluorescent signals reached a plateau, the endpoints of the reaction were used for analysis.

\section{BRD inhibition assay}

BET inhibition was measured using the AlphaScreen assay. The reagents were diluted with buffer (0.1\% BSA, $50 \mathrm{mM}$ HEPES, and 100 $\mathrm{mM} \mathrm{NaCl}$ ) supplemented with $0.05 \%$ CHAPS. Four milliliters of protein were added to 384-well plates, followed by nonbiotinylated peptides, solvents, or compounds. The plates were then incubated at $25^{\circ} \mathrm{C}$ for $25 \mathrm{~min}$ and then $4 \mathrm{~mL}$ of biotinylated peptides were added. The plates were resealed and incubated for another $25 \mathrm{~min}$. Four microliters of nickel chelate AlphaLISA Acceptor beads and streptavidin-coated donor beads were then added. The plates were incubated and luminescence was detected using a PHERA star FS plate reader and the AlphaScreen 570 emission/680 excitation filter set.

\section{MTT assay}

$7.0 \times 10^{3}$ cells per well were seeded into 96-well plates, incubated and then treated with various concentrations of compounds and incubated at $37^{\circ} \mathrm{C}$ for $48 \mathrm{~h}$. Then, $30 \mu \mathrm{L}$ MTT solution were added and cultured, and the crystals of formazan product were then dissolved by oscillating for 10 min. The optical density (OD) value was detected using a microplate reader (Bio-Rad, imark, Hercules, CA) at a wavelength of $570 \mathrm{~nm}$. The value of inhibitory concentration $50\left(\mathrm{IC}_{50}\right)$ was calculated by GraphPad Prism 5 software (La Jolla, CA).

\section{Cell culture}

All human breast cancer cell lines (MCF-7, MDAMB-438, MDA-MB-435, and MDA-MB-231) were obtained from the CBTCC of the Chinese Academy of Sciences (Shanghai, China). Cells were cultured in RPMI-1640, F12, or DMEM supplemented with fetal bovine serum (10\% FBS, Hyclone, Logan, UT, USA) and penicillin/streptomycin (1\%), and maintained in in an incubator $\left(5 \% \mathrm{CO}_{2}\right.$ at $\left.37^{\circ} \mathrm{C}\right)$. 


\section{Western blot analysis}

Cells were incubated with various concentrations of chidamide, PFI-1, or a combination of chidamide and PFI-1 for $48 \mathrm{~h}$. Cells were collected and lysed in cold lysis buffer for total protein extraction. Protein samples $(15 \mu \mathrm{g})$ were electrophoresed on an SDS-polyacrylamide gel and transferred to a polyvinylidene difluoride (PVDF) membrane, and then incubated with primary specific antibodies against p-STAT3 and glyceraldehyde phosphate dehydrogenase (GAPDH). After washing, membrane was incubated with the secondary antibody for $2 \mathrm{~h}$ and determined by chemiluminescence.

\section{In vivo studies}

For in vivo studies, MDA-MB-231 cells $\left(1 \times 10^{6}\right)$ were diluted and injected subcutaneously into 5week-old female athymic nude mice (18-22 g). Three days later, the mice were divided randomly into four groups: (1) vehicle gavage (saline containing $4 \%$ DMSO, $5 \%$ PEG, and $5 \%$ Tween 80; once every 3 days for 18 days); (2) chidamide or (3) PFI-1 gavage (100 mg/kg compound, once every 3 days for 18 days); or (4) chidamide and PFI-1 combination gavage ( 50 $\mathrm{mg} / \mathrm{kg} /$ compound, once every 3 days for 18 days). The tumor volumes were measured once every 3 days as length $\times$ width $\times$ height (in $\mathrm{mm}^{3}$ ). Meanwhile, mouse body weights were measured and recorded. All studies were approved by the Animal Ethics Committee of First Affiliated Hospital of Xiamen University, and the experiments were carried out in accordance with the guidelines of Guide for the care and use of laboratory animals[15].

\section{Immunohistochemistry (IHC) staining assay}

Paraffin-embedded tumor xenografts were sectioned, and slides were prepared and immunostained using primary antibodies against p-STAT3. Immunohistochemistry (IHC) labeling was conducted using $0.1 \%$ trypsin for antigen retrieval using the immunoperoxidase method. Slides were then stained with dye followed by dehydration. Slides were scanned, and images were obtained using an x-cite 120 (Olympus) microscope.

\section{Statistical analysis}

The experiments were performed in triplicate. Statistical significance was evaluated using the Student's $t$ test for multiple comparisons using GraphPad Prism software $\left\{p<0.001{ }^{\left({ }^{* \star}\right)} ; p<\right.$ $0.01\left(^{* *}\right)$; and $\left.p<0.05\left(^{*}\right)\right\}$.

\section{RESULTS}

\section{Affinity and binding of PFI-1 and chidamide}

The inhibitory effects of chidamide and PFI-1 on HDACs and BET proteins were examined, respectively. By detecting the enzymatic activities of human recombinant histone deacetylases (HDACs), the efficiency of the competitive binding of chidamide to HDAC subtypes in vitro was profiled. As shown in Figure $1 \mathrm{C}$, chidamide inhibited the enzymatic activities of HDAC1, HDAC2, and HDAC3 at low nanomolar concentrations, which was consistent with previous study [16]. The $I_{50}$ values of chidamide for HDAC1, HDAC2, and HDAC3 were $55.91 \mathrm{nM}, 738.08 \mathrm{nM}$, and $61.64 \mathrm{nM}$, respectively. The binding model between chidamide and HDAC was predicted through a docking study. As shown in Figure 2 A, chidamide bound tightly to HDAC via a $\pi-\pi$ interaction between Phe155 and Phe210, and the zinc ion was chelated by the carbonyl group of the benzamide.

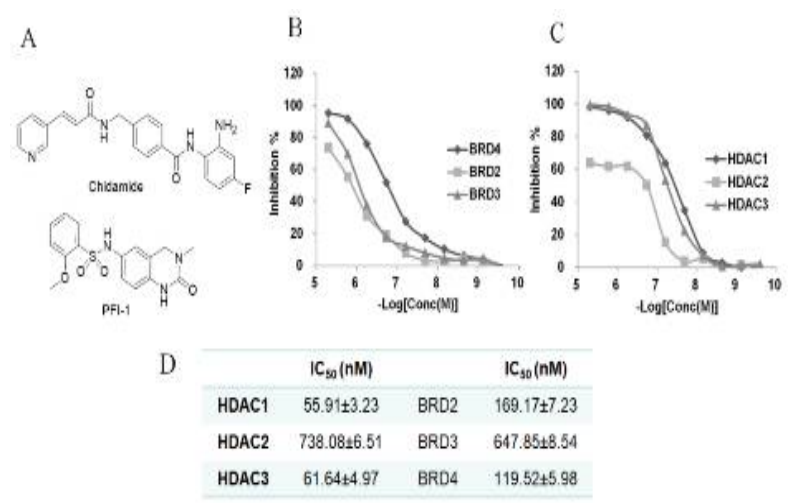

Figure 1: The inhibitory effects of chidamide on HDACs and and PFI-1 on BET proteins. (A) Chemical structures of chidamide and PFI-1. (B) AlphaScreen assay dose-response curves, displaying the replacement of bromodomains BRD2, BRD3, and BRD4 with acetylated lysine residues by PFI-1. (C) Dose-response curves showed the inhibitory effects of chidamide on the enzymatic activities of HDAC1, HDAC2, and HDAC3. (D) $I C_{50}$ values were determined ranging from $5.00 \times 10^{-6}$ to $2.54 \times 10^{-10} \mathrm{M}$

Similarly, PFI-1 inhibited BET proteins (BRD4, BRD3, and BRD2) at submicromolar concentrations (Figure $1 \mathrm{~B}$ and $\mathrm{C}$ ). $I \mathrm{IC}_{50}$ values for $\mathrm{BRD2}$, BRD3, and BRD4 were $169.17 \mathrm{nM}$, $647.85 \mathrm{nM}$, and $119.52 \mathrm{nM}$, respectively. A cocrystal structure was obtained for $\mathrm{PFI}-1$ binding to BRD4 by Fish et al [17]. Figure 2 B demonstrated that PFI-1 bound tightly to BRD4. The urea carbonyl and $\mathrm{NH}$ group interacted with Asn140. 

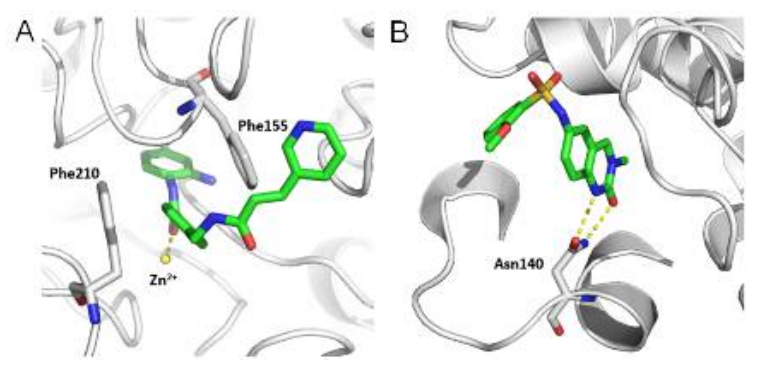

Figure 2: Three-dimensional model of the interactions between chidamide and HDAC2 and between PFI-1 and BRD4. (A) The interaction between chidamide and HDAC2 (PDB ID 4LXZ). Chidamide is depicted as sticks with carbon atoms in green, oxygen in red, and nitrogen in blue. The yellow sphere represents the zinc ion, and the yellow dashed lines indicate chelation. (B) The X-ray crystal structure of interaction between PFI and BRD4 (PDB ID 4E96). The hydrogen bond is denoted by yellow dashed lines. The color code is the same as in A. Figures were prepared using PyMOL Molecular Graphics System

\section{Inhibitory effect of chidamide or PFI-1 on breast cancer cell proliferation in vitro}

Based on these interactions, the effects of chidamide or $\mathrm{PFI}-1$ treatment on four breast cancer cell lines (MCF-7, MDA-MB-438, MDAMB-435, and MDA-MB-231) were performed. 5 $\mu \mathrm{M}$ of chidamide or $5 \mu \mathrm{M} \mathrm{PFI-1}$ was treated and the cell viability was determined. As shown in Figure 3 , the percentage of the viability of four breast cancer cell lines were significantly decreased by chidamide or PFI-1 treatment than that treated with DMSO.

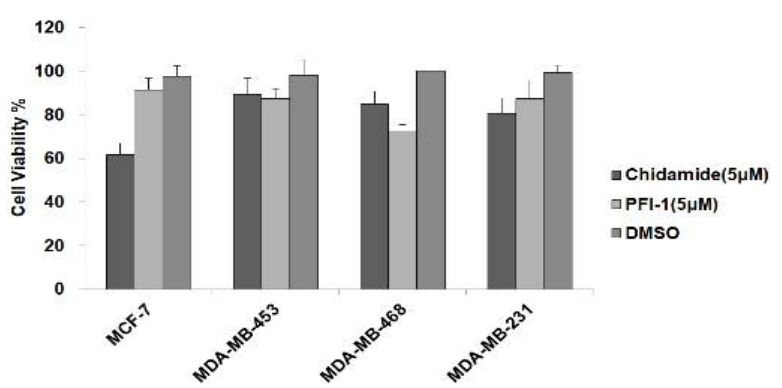

Figure 3: Effect of chidamide or $\mathrm{PFI}-1$ on the viability of breast cancer cell lines. MCF-7, MDA-MB-453, MDA-MB-468, and MDA-MB-23 cells were seeded into 96-well plates and treated for $48 \mathrm{~h}$ with chidamide (5 $\mu \mathrm{M})$ or PFI-1 $(5 \mu \mathrm{M})$, and evaluated using the MTT assay

Inhibitory effect of the combination of PFI-1 and chidamide on the viability of MDA-MB231 cells

Among four breast cancer cell lines, a TNBC cell line MDA-MB-231 showed the lowest sensibility to either chidamide or PFI-1, was selected which was used to further evaluate the comprehensive effects of PFI-1 and chidamide. T As shown in Figure $4 \mathrm{~A}$ to $\mathrm{C}$, the combination of PFI-1 and chidamide could significantly decrease the cell viability of MDA-MB-231 cells as compared to that treated with $\mathrm{PFI}-1$ or chidamide alone. Besides, this inhibitory effect was dosedependently.

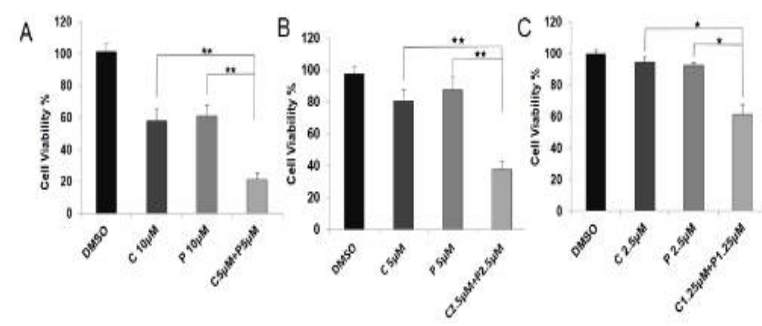

Figure 4: The inhibitory effects of the combination of $\mathrm{PFI}-1$ and chidamide on the viability of MDA-MB-231 cells MDA-MB-231 cells were seeded into 96-well plates and treated for $48 \mathrm{~h}$ with various concentrations of chidamide alone, PFI-1 alone, or the combination of PFI-1 and chidamide, and was evaluated using the MTT assay. (A) Chidamide $(10 \mu \mathrm{M})$, PFI-1 $(10 \mu \mathrm{M})$, and chidamide $(5 \mu \mathrm{M})+\mathrm{PFI}-1(5 \mu \mathrm{M})$. (B) Chidamide $(5 \mu \mathrm{M}), \mathrm{PFI}-1(5 \mu \mathrm{M})$, and chidamide $(2.5 \mu \mathrm{M})+\mathrm{PFI}-1$ $(2.5 \mu \mathrm{M})$. (C) Chidamide $(2.5 \mu \mathrm{M})$, PFI-1 $(2.5 \mu \mathrm{M})$, and chidamide $(1.25 \mu \mathrm{M})+\mathrm{PFI}-1(1.25 \mu \mathrm{M})$. C: Chidamide, $\mathrm{P}: \mathrm{PFI}-1 ; p<0.05\left(^{*}\right) ; p<0.01\left(^{* *}\right)$

Effects of the combination of PFI-1 and chidamide on protein expression of P-STAT3 in MDA-MB-231 cells

To verify whether STAT3 phosphorylation ( $p$ STAT3) was involved in the synergistic effects of PFI-1 and chidamide on MDA-MB-231 cells, the protein expression of STAT3 phosphorylation ( $p$ STAT3) was detected using western blot analysis. As shown in Figure 5, the combination of $\mathrm{PFI}-1$ and chidamide could significantly decrease the protein expression of p- STAT3 in MDA-MB-231 cells as compared to that treated with PFI-1 or chidamide alone. Besides, this inhibitory effect was dose-dependently.

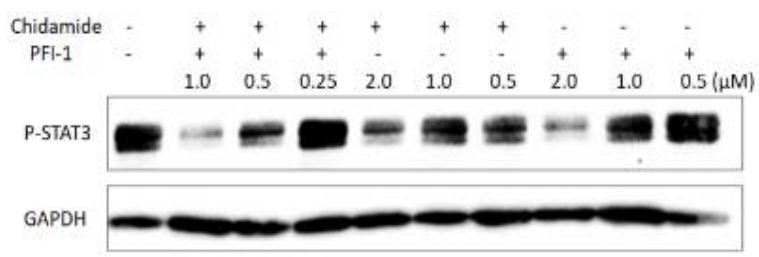

Figure 5: Effect of the combination of PFI-1 and chidamide on the protein expression of p-STAT3 in MDA-MB-231 cells. Immunoblot analysis of p-STAT3 and GAPDH after treated with DMSO, chidamide, PFI1 , or a combination of both chidamide and PFI-1 in MDA-MB-231 cells for 48h. "+" refers to with treatment, "-" refers to without treatment 
Inhibitory effect of the combination of PFI-1 and chidamide on tumor growth in vivo

The therapeutic efficacy of chidamide and PFI-1 was assessed in a subcutaneous human breast cancer xenograft (MDA-MB-231 cell) model. As shown in Figure $6 \mathrm{~A}$, treatment with either chidamide $(100 \mathrm{mg} / \mathrm{kg}$ ) or PFI-1 (100 mg/kg) alone had limited effects. Nevertheless, treatment with a combination of chidamide and PFI-1 elicited a synergistic effect on tumor growth, showing lower tumor volume as compared to that treated with chidamide or PFI-1 alone. In addition, histochemical staining of $p$ STAT3 demonstrated that its levels were suppressed by the combination treatment of chidamide and $\mathrm{PFI}-1$ in vivo, as compared to that treated with chidamide or PFI-1 alone (Figure 6 B). However, there was no observable difference in mouse weight among groups, indicating that this combination therapy was tolerated by mice (Figure 6 C).

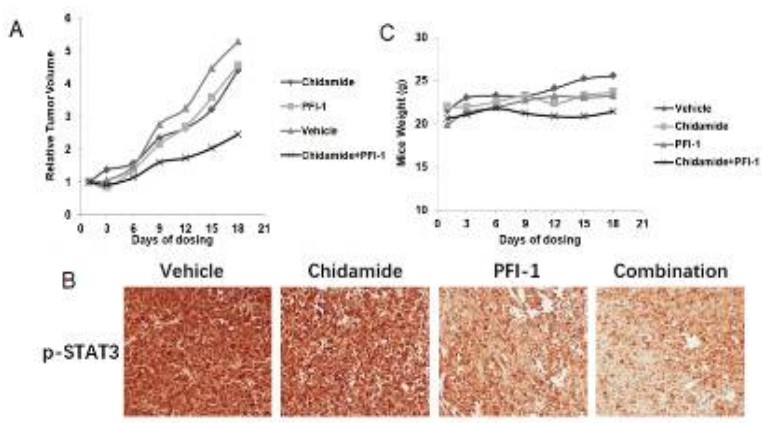

Figure 6: The inhibitory effects of the combination of PFI-1 and chidamide on tumor growth in vivo. (A) TNBC mouse model were constructed and were treated with chidamide (100 mg/kg), PFI-1 (100 $\mathrm{mg} / \mathrm{kg}$ ), or chidamide (50 mg/kg) + PFI-1 (50 mg/kg) for 21 days. Tumor growth curves were plotted by measuring the relative tumor volume once every 3 days. (B) The protein expression of STAT3 phosphorylation ( $p$ - STAT3) in the TNBC mouse model was assessed by immunohistochemical analysis. (C) Mice were treated with chidamide (100 $\mathrm{mg} / \mathrm{kg}$ ), PFI-1 (100 mg/kg), or chidamide (50 mg/kg) + PFI-1 (50 mg/kg) for 21 days. Mouse body weights were measured once every 3 days

\section{DISCUSSION}

Chidamide is a novel, small molecule and $\mathrm{HDACi}$ is characterized by a benzamide scaffold group (Figure 1 A) [16]. It was developed by Shenzhen Chipscreen Biosciences Ltd. and approved by the Chinese FDA in 2015 for relapsed or refractory peripheral T-cell lymphoma. PFI-1 (Figure $1 \mathrm{~A}$ ) is a selective BETi that blocks cellular proliferation in leukemic cell lines [18]. In our study, chidamide had inhibitory effects on HDACs and PFI-1 had inhibitory effects on BET proteins. The three-dimensional model showed the interactions between chidamide and HDAC2 and between PFI-1 and BRD4. The results in our study indicated that chidamide alone or PFI-1 alone elicits inhibitory effects on breast tumor cell viability. However, the combination of PFI-1 and chidamide could significantly inhibit MDA-MB231 cell viability, and increase the expression of p-STAT3, as compared to that treated with chidamide or PFI-1 alone. Moreover, the inhibitory effects of PFI-1 and chidamide on tumor growth were also found in the in vivo mice experiments. These results indicated that chidamide and PFI-1 could, at least partially, inhibit the progression and development of TNBC. Recent studies have suggested that the inhibition of STAT3 activation acts as an effective TNBC therapy [19-22]. While single treatments of either PFI-1 or chidamide suppressed STAT3 phosphorylation at concentrations less than 2.0 $\mu \mathrm{M}$ (mainly by PFI-1), the combination treatment caused significant reduction in the protein level of p-STAT3. These findings suggested that the inhibition of STAT3 activation may block the progression and development of TNBC. Thus, chidamide and PFI-1 may inhibit the progression and development of TNBC by decreasing $p$ STAT3 expression.

\section{CONCLUSION}

The distinct effects of chidamide, PFI-1 and a combination of chidamide and PFI-1, on the cell viability of TNBC both in vitro and in vivo have been investigated comprehensively. The combination of chidamide and PFI-1 inhibits cell viability in vitro and suppresses tumor growth in vivo when compared to treatment with chidamide, or $\mathrm{PFI}-1$ alone. Thus, the combination therapy is a potential therapy for the management of TNBC.

\section{DECLARATIONS}

\section{Conflict of interest}

No conflict of interest is associated with this work.

\section{Contribution of authors}

We declare that this work was done by the researchers listed in this article. All liabilities related with the content of this article will be borne by the authors. Nong Lin designed all the experiments and revised the paper. Qiaolu Yang and Tong $\mathrm{Xu}$ formed the experiments, while Lianguo Shi wrote the paper. 


\section{Open Access}

This is an Open Access article that uses a funding model which does not charge readers or their institutions for access and distributed under the terms of the Creative Commons Attribution License (http://creativecommons.org/licenses/by/ 4.0) and the Budapest Open Access Initiative (http://www.budapestopenaccessinitiative.org/rea d), which permit unrestricted use, distribution, and reproduction in any medium, provided the original work is properly credited.

\section{REFERENCES}

1. Qiu JD, Xue $X Y$, Li R, Wang JD. Clinicopathological features and prognosis of triple-negative breast cancer: a comparison between younger $(<60)$ and elderly $(>/=60)$ patients. Eur J Cancer Care (Engl) 2016; 25(6): 1065-1075.

2. O'Shaughnessy JA. Breast cancer in focus: treatment options for triple-negative metastatic breast cancer. Clin Adv Hematol Oncol 2012; 10(1): 43-45.

3. Andergassen U, Kolbl AC, Mumm JN, Mahner S, Jeschke $U$. Triple-negative breast cancer: New therapeutic options via signalling transduction cascades. Oncol Rep 2017; 37(5): 3055-3060.

4. Bilici A, Arslan C, Altundag K. Promising therapeutic options in triple-negative breast cancer. J BUON 2012; 17(2): 209-222.

5. Gucalp A, Traina TA. Triple-negative breast cancer: adjuvant therapeutic options. Chemother Res Pract 2011; 2011: 1-13.

6. Qin C, Hu Y, Zhou B, Fernandez-Salas E, Yang CY, Liu L, McEachern D, Przybranowski S, Wang M, Stuckey J et al. Discovery of QCA570 as an Exceptionally Potent and Efficacious Proteolysis Targeting Chimera (PROTAC) Degrader of the Bromodomain and ExtraTerminal (BET) Proteins Capable of Inducing Complete and Durable Tumor Regression. J Med Chem 2018; 61(15): 6685-6704.

7. Borah N, Gunawardana S, Torres $H$, McDonnell S, Van Slambrouck S. 5,6,7,3',4',5'-Hexamethoxyflavone inhibits growth of triple-negative breast cancer cells via suppression of MAPK and Akt signaling pathways and arresting cell cycle. Int J Oncol 2017; 51(6): 1685-1693.

8. Ma X-t, S-hR, Y-cZ, L-tJ. Current perspectives on genotype classification and individualized drug targeting in triple-negative breast cancer. Tropical Journal of Pharmaceutical Research 2018; 2(17): 359-364.

9. Di Pietro A, Good-Jacobson KL. Disrupting the Code: Epigenetic Dysregulation of Lymphocyte Function during Infectious Disease and Lymphoma Development. J Immunol 2018; 201(4): 1109-1118.

10. Mardin WA, Haier J, Mees ST. Epigenetic regulation and role of metastasis suppressor genes in pancreatic ductal adenocarcinoma. BMC Cancer 2013; 13:264-272.
11. Rose S. HDAC inhibitors show benefits in breast cancer. Cancer Discov 2011; 1(7): OF1-2.

12. Vrba J, Trtkova K, Ulrichova J. HDAC inhibitors sodium butyrate and sodium valproate do not affect human ncor1 and ncor2 gene expression in HL-60 cells. Biomed Pap Med Fac Univ Palacky Olomouc Czech Repub 2011; 155(3): 259-262.

13. Denis GV, Nikolajczyk BS, Schnitzler GR. An emerging role for bromodomain-containing proteins in chromatin regulation and transcriptional control of adipogenesis. FEBS Lett 2010; 584(15): 3260-3268.

14. Badamchi-Zadeh A, Moynihan KD, Larocca RA, Aid M, Provine NM, lampietro MJ, Kinnear E, PenalozaMacMaster $P$, Abbink $P$, Blass $E$ et al. Combined HDAC and BET Inhibition Enhances Melanoma Vaccine Immunogenicity and Efficacy. J Immunol 2018; 201(9): 2744-2752.

15. Care IoLARCo, Animals UoL: Guide for the care and use of laboratory animals: US Department of Health and Human Services, Public Health Service, National Institutes of Health; 1986.

16. Ning $Z Q$, Li ZB, Newman MJ, Shan S, Wang $X H$, Pan $D S$, Zhang J, Dong M, Du X, Lu XP. Chidamide (CS055/HBI-8000): a new histone deacetylase inhibitor of the benzamide class with antitumor activity and the ability to enhance immune cell-mediated tumor cell cytotoxicity. Cancer Chemother Pharmacol 2012; 69(4): 901-909.

17. Barciszewska A-M. MicroRNAs as efficient biomarkers in high-grade gliomas. Folia Neuropathol 2016; 54(4): 369374.

18. Fish PV, Filippakopoulos $P$, Bish $G$, Brennan $P E$, Bunnage ME, Cook AS, Federov O, Gerstenberger BS, Jones $H$, Knapp $S$ et al. Identification of a chemical probe for bromo and extra C-terminal bromodomain inhibition through optimization of a fragment-derived hit. J Med Chem 2012; 55(22): 9831-9837.

19. Chang $R$, Song $L, X u Y$, Wu Y, Dai C, Wang $X$, Sun $X$, Hou $Y$, Li W, Zhan $X$ et al. Loss of Wwox drives metastasis in triple-negative breast cancer by JAK2/STAT3 axis. Nat Commun 2018; 9(1): 3486.

20. Dai X, Yin C, Guo G, Zhang Y, Zhao C, Qian J, Wang O, Zhang $X$, Liang $G$. Schisandrin $B$ exhibits potent anticancer activity in triple negative breast cancer by inhibiting STAT3. Toxicol Appl Pharmacol 2018; 358:110-119.

21. Liu J, Liu L, Yague E, Yang Q, Pan T, Zhao H, Hu Y, Zhang J. GGNBP2 suppresses triple-negative breast cancer aggressiveness through inhibition of IL-6/STAT3 signaling activation. Breast Cancer Res Treat 2019; 174(1): 65-78.

22. Pindiprolu S, Chintamaneni PK, Krishnamurthy PT, Ratna Sree Ganapathineedi K. Formulation-optimization of solid lipid nanocarrier system of STAT3 inhibitor to improve its activity in triple negative breast cancer cells. Drug Dev Ind Pharm 2019; 45(2): 304-313. 